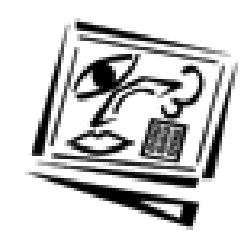

Australian Journal of
Educational Technology

\title{
Developing effective hypermedia instructional materials
}

\author{
Ron Oliver \\ Edith Cowan University \\ r.oliver@cowan.edu.au \\ Jan Herrington \\ Edith Cowan University
}

\begin{abstract}
The use of hypermedia as an instructional medium is growing rapidly with developments and enhancements in instructional and computing technologies. Much of the popularity of the medium is derived from its capacity to convey large amounts of information to learners in structured and associated ways which promote student centred and independent learning. Recent developments in multimedia technologies and software, and enhanced networking facilities through the Internet, have led to new and exciting opportunities for hypermedia systems development. This paper provides an overview of the potential of hypermedia as an instructional medium and discusses research that has investigated learning outcomes. It provides an overview of instructional design considerations to guide developers and designers of hypermedia systems.
\end{abstract}

Hypertext, or hypermedia as it is more commonly called today, is an instructional medium which appears to hold considerable promise for teaching and learning. It manifests itself in many forms, often with quite disparate appearances and capabilities. For example, the majority of $\mathrm{CD}$ ROM based information systems such as electronic encyclopedia are examples of hypermedia systems as are the majority of instructional materials delivered on the World Wide Web. Although different in appearance, scope and form, the unifying characteristics of these computer based materials are the structure they embrace and the purpose they serve.

Structure. Hypermedia materials are comprised of multiple separate information nodes. These information nodes contain various media forms such as text, sound, graphics and movies either individually or combined. The structure of a hypermedia system enables users to access information from the nodes in a non linear way. Users are able to progress from one node to the next using links supplied by the system designer. The two 
fundamental units in a hypermedia system are the information nodes themselves and the links that connect them.

The links in a hypermedia system create associations between the nodes. Links are usually provided only between nodes that are associated or semantically related. The structure of a hypermedia system is often compared to the human mind which also stores information as discrete nodes with links between those that are related and/or associated.

Purpose. Hypermedia materials are usually designed as information delivery systems. In this role they serve an instructional purpose. While some systems are designed as information sources within which the user is free to browse, others constrain the user to follow paths through the stored information. Such paths are set by the instructional designer and lead the user through the information in the same way as a book presents content to a reader. As purveyors of information, hypermedia systems provide many advantages over paper based forms. They are very flexible and powerful in the way in which information is accessed and provide access to multiple media forms.

Nature. Hypermedia systems employ many consistent features in the way in which they are designed and used. In the first instance, the systems are comprised of large amounts of information. It is not uncommon for hypermedia systems on $\mathrm{CD}$ ROM to provide access to the equivalent amount of information as would be found in dozens of books. Network based systems, such as the World Wide Web, have an almost unlimited scope for information storage. The stored information is predominantly text supported by graphical images, photographs, sounds, animations and movies. Navigation among the nodes is relatively simple. Related nodes are usually identified within a node by the use of typographic cues such as underlining or emboldening. Links are usually made by single mouse clicks on keywords or cues. New nodes are displayed in the form of windows that overlay. Closing or selecting windows provides a means of moving back to previously visited nodes.

The potential of hypermedia as a learning tool is derived from the nature of the learning that it supports. It facilitates student centred approaches, creating a motivating and active learning environment (Becker \& Dwyer, 1994). It supports and encourages browsing and exploration, learner behaviours that are frequently associated with higher order learning (Thuring, Mannemann \& Haake, 1995). The nature of information organisation in hypermedia appears to closely mimic human memory. Information retrieval methods closely resemble human thought processes. People do not think using indexing and sorting rules but rather in terms of contextual links between information and images. Hypermedia supports and facilitates a very natural and efficient form for information retrieval (Dimitroff \& Wolfram, 1995). These and other advantages offered by the 
medium have created considerable enthusiasm among learning theorists and teachers towards hypermedia as an effective learning tool. The reality, however, reveals a number of constraints that can limit its effectiveness in general use.

\section{Teaching and Learning with Hypermedia}

As with the majority of computer based learning materials, hypermedia systems tend to be developed around text based nodes and instructional guides. Often there is an implicit assumption made in developing text based instructional materials that, through the act of reading, learning will be achieved. Usually when text based instructional materials are created, they are developed through a process where relevant ideas and concepts are presented in a particular sequence and fashion (Herrington, Fox, Gillard \& Rainford, 1992). When a student reads and interacts with materials of this form, there are a number of factors that influence how this information is processed and ultimately what is learned. These factors include:

- the nature and scope of the material to be learned;

- how the content is presented; and

- the way it is processed by the individual learner.

The nature of the material to be learned. While text based descriptions can be used effectively to impart low level procedural knowledge, it is more difficult to use this medium for declarative and higher knowledge levels. Knowledge that is comprised of facts, procedures and rules of discourse is usually taught and learned in ways where an instructor plays a significant role in content delivery (Jonassen, Mayes \& McAleese, 1993). This form of instruction is frequently used to facilitate initial knowledge acquisition in a field. But it is less effective when used as a means to present advanced knowledge where principles and concepts need to be developed. Contemporary educational theory suggests that higher order learning is best achieved through instructional processes that support student centred, collaborative and generative activity. Such instruction places the learner at the centre of the teaching and learning process and active in constructing a personal meaning of the content being delivered (Knuth \& Cunningham, 1993). When higher order learning goals are sought, the forms of learning environments that are most effective are those which:

- provide multiple representations of reality;

- focus on knowledge construction rather than knowledge transmission;

- present authentic tasks that foster reflective practice;

- enable context and content dependent knowledge construction; and

- support collaborative construction of knowledge through social negotiation rather than competition (Jonassen, 1994). 
These forms of learning environment are not usually seen or associated with learning based on instructional texts although there are strategies by which they can be implemented in text (eg. Herrington, Fox, Gillard \& Rainford, 1992). As an instructional medium, however, hypermedia appears to hold considerable potential to reverse this trend. It provides a means for developing instructional materials that can be used for student centred activities that students find motivating and appealing (Becker \& Dwyer, 1994).

Content presentation. There are many ways in which text based materials can be presented to the learner. Exactly the same material can be presented in a myriad of ways. Variations include such items as layout, organisation, and structure and appearance. For learning to result, a student must be able to comprehend what is being read. Thuring, Mannemann \& Haake (1995) suggest that the principle elements to be considered in presentation of instructional text are structure and readability. Both of these are considered to significantly influence learner's comprehension. Comprehension is characterised as the construction of a mental model that represents the objects and semantic relations described in a text (van Dijk \& Kintsch, 1983). In developing comprehension of material, a student builds a mental representation of the contents of a document. This mental representation involves linking small pieces of information together to form larger chunks in the form of a hierarchical macrostructure (Thuring, Mannemann \& Haake, 1995). Text is more readily comprehended by a reader when it is coherent, that is, when it presents the information in a way that facilitates linking between discrete elements and the chunking of linked elements into larger items.

The very nature of hypermedia as a set of connected information nodes provides and supports the construction of coherent and well structured instructional materials.

Learner characteristics. The characteristics of individual learners also play a significant role in determining how much is learned from hypermedia and hypertext (Marchionini, 1989; Riding \& Chambers, 1992). Hypertext and hypermedia environments have been found to cater for individual learner differences by allowing learners some control over how long is spent on particular tasks and within certain topics enabling them to choose when to proceed and when to pause (Small \& Grabowski, 1992). At the same time, such personal attributes as metacognitive skills and levels of self regulation can also influence the level of learning achieved.

Metacognition describes a learner's understanding and knowledge of how he or she is learning and what can be done to influence this (Puntambekar, 1995). There are two important aspects of metacognition. The first is an awareness about cognition and learning and the second is control or regulation of that learning (Brown, 1978). Learners display metacognition 
when they are able to judge what is being learned and instigate actions and processes to overcome perceived shortcomings (Balajthy, 1990). Self regulation is described by Kunz, Drewniak \& Schott ( 1992) as the goal directed, controllable and reflective cognitive activities a learner uses to enhance learning. Learners vary considerably in the level of self regulation that they exhibit. A high level of self regulation involves such activities as strategic implementation, sequencing of goal adaptive strategies, flexible planning, monitoring of ongoing information processing and the intelligent evaluation of ones' own cognitive endeavours. Strong learners often demonstrate sound knowledge of their own cognitive resources and an understanding of the connections between the task at hand and the possible strategies able to be used in the context of the cognitive endeavour (Kunz, Drewniak \& Schott, 1992). On the other hand learners with low self regulation ability are frequently found to be less successful in learning tasks as a consequence of such actions as:

- failing to integrate new knowledge with prior knowledge;

- using rigid criteria to monitor their own reading comprehension; and

- forming conceptual misunderstandings at post introductory stages of learning (Jacobson \& Spiro, 1995).

Hypermedia materials can be constructed to provide strong support for self regulation and metacognition among users. A number of models and strategies of instructional support have been designed and successfully implemented within hypermedia systems to facilitate self regulation and goal based activity among users (eg. Cheng \& Rowe, 1995).

\section{Hypermedia Forms}

While hypermedia describes a particular type of learning environment, there are many forms of exposition that assume this title (Gillingham, 1993). A useful way to discuss and compare the different forms of hypermedia is through a continuum describing the nature of the linking involved. At one end of the continuum, the links are minimal and simply act to connect nodes in a specified sequence. This form of hypermedia closely resembles conventional text and is referred to as linear. In its use; the reader is encouraged and in most cases compelled to follow an instructional sequence planned by the instructor. In hypermedia environments, there is potential to create materials with varying degrees of linearity. Further along the continuum, the links tend to form a hierarchical structure, giving learners more freedom in the choice of path through the materials. At the extreme, hypermedia can provide a totally unstructured learning environment with multiple links between associated nodes. In this environment, learners are free to move between associated nodes through referential links and very little structure is evident. This is the pure form of hypermedia, the form that most closely 
resembles the stated definition. Figure 1 shows the continuum and the relative position of each of the forms of linking supported.

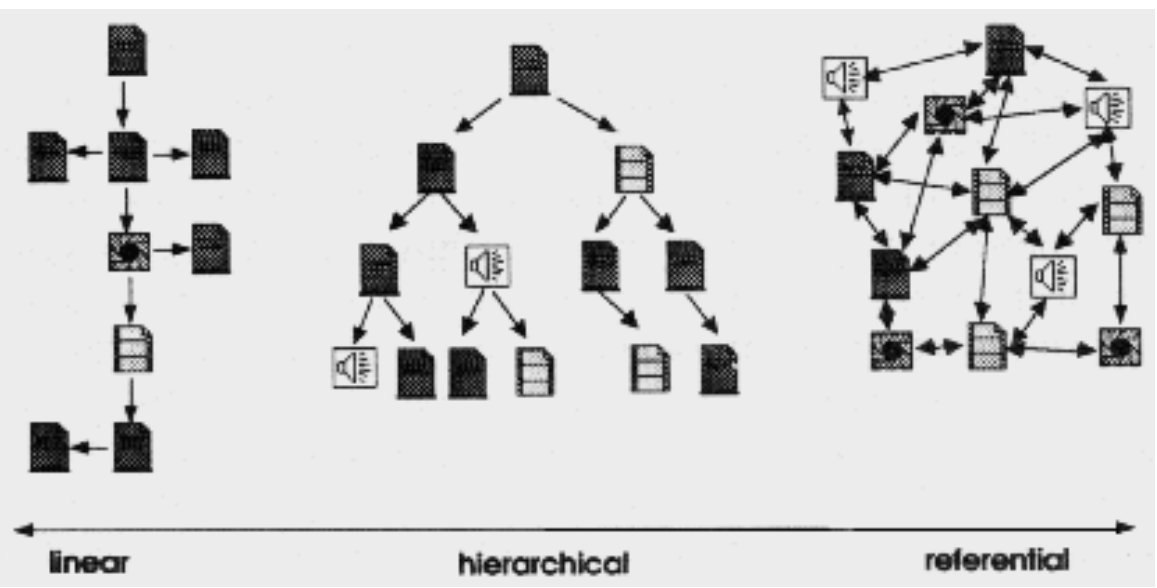

Figure 1: A continuum describing linking in hypermedia.

Most materials developed for World Wide Web publication are of the linear form. The materials typically follow a planned sequence with links to other sites and nodes from among which the learner can choose. The student is expected and encouraged to visit these sites while the overall structure of the sequence is provided by adherence to a main document. Hierarchical links are common in electronic encyclopedia as one of the many navigation forms supported. Learners make decisions as they move through a less structured environment seeking information. There is no set path and often the same learning outcomes can be achieved by travelling different paths. There are now a number of mediabases that represent good examples of hypermedia with referential links. The Australian product Investigating Lake Iluka (1995) is one such case. This program provides access to a myriad of information nodes with linking that provides little constraint. Learners can move freely among the nodes in any sequence and this feature of the program is seen as a major strength.

In designing hypermedia materials, an early decision that must be taken relates to the nature of the linking that will be used. This decision must be based on such considerations of the forms of learning outcomes required and the nature of the learners. Many writers argue that instructional text needs to have a defined structure and that providing students with the means to avoid the structure can limit the learning that is achieved. Some writers argue that many students do not have the capacity to choose the best path through the instructional materials and that the freedom provided by a hypermedia environment can compromise the 
responsibility of the instructional designer to guide the learner (eg. Trumbull, Gay \& Mazur, 1992; McGrath, 1992; Glover, 1994).

Even when hypermedia is designed for particular uses and applications, it is not always used as it was intended (Heller, 1990). Studies have revealed large differences in the way individuals manage their own reading and learning strategies. Because some learners exhibit poor self regulation and metacognition when reading from instructional text, they find it difficult to accurately monitor their own success or failure in learning. This problem is also evident in most computer based learning situations where some form of control of the learning environment is given to the learner. For example, Balajthy (1990) observed a tendency among some users of a hypertext system to terminate instruction prematurely not knowing whether the learning task has been achieved. Piette and Smith (1991) describe a study of hypertext usage where students took advantage of the high levels of user control and took shortcuts in the instructional sequence, often to the detriment of their learning. A second version of the hypermedia environment containing less learner control was developed. Implementation of the second version revealed more effective learning was achieved in this teacher controlled environment.

A number of projects have made specific attempts to guide and direct users through the use of computer intelligence to monitor and lead learners through the hypermedia environment. In one such system, an intelligent guide was programmed to respond to previous actions of the users in the hypertext system and to adjust instructional screens to provide extra information and advice where and when it was judged to be needed. Usage patterns, however, did not always reflect expectations. Observations of users revealed that many ignored these instructions and misused advice when it conflicted with their personal goals, knowledge and experience (Carroll, 1990).

The decision of the scope and level of user control, and as a consequence the form of hypermedia implementation, is often difficult to make. The work of Jonassen, Mayes \& McAleese (1993) provides a useful guide for selecting the form of hypermedia most suited to the nature of intended learning outcomes by suggesting instructional strategies against knowledge acquisition aims (Figure 2). When the instructional forms of the different forms of hypermedia are matched against the continuum describing instructional strategies, it is evident that the development of procedural knowledge, for example, facts, procedures and rules of discourse, linear linking would be an appropriate hypermedia form. For higher levels of knowledge (such as developing an understanding of concepts and principles), the less structured hierarchical and referential linkings are more appropriate. 


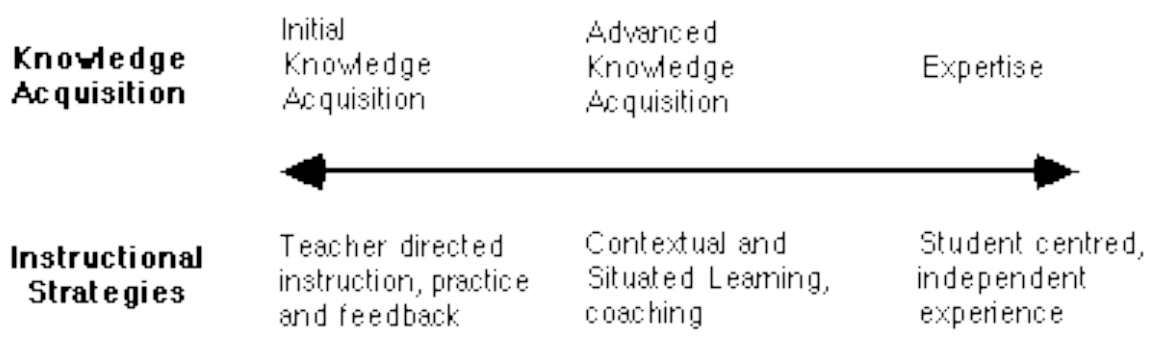

Figure 2: A continuum of knowledge acquisition and facilitative strategies.

While the choice of linking for hypermedia is based primarily on intended learning, it is at this stage that the target audience needs also to be considered. The different forms of linking place different expectations on the role of the learner in the instructional process. Linear linking involves the lowest levels of learner decision making and control. When materials are prepared in this form, the instructor is able to exert the greatest influence on the information that will be accessed by the learners. With the less structured hierarchical and referential forms of linking, the instructor usually guides the learner by providing an external context for use of the hypermedia. For example, learners may be asked to use the hypermedia system as an information source in order to solve a specific problem or series of problems. Learners might be encouraged to work cooperatively and collaboratively with the hypermedia system being a tool for their inquiry and investigation.

Computer based activities of this kind are frequently described as those capable of supporting higher order learning outcomes (eg. Young, 1995; Herrington \& Oliver, 1995). Jacobson \& Spiro (1995) report a study which compared learning achieved through use of conventional computer based instruction and a hypermedia system. A control group were exposed to computer based instruction which presented material in a linear and sequential form and used drill and practice to test achievement. The experimental group had access to a hypermedia system which presented the same information in the form of a series of cross linked thematic nodes. In achievement tests, the students who learned from the linear approach achieved higher scores in tests of memory while those exposed to the hypermedia system scored significantly higher in tests of higher order cognitive skills such as knowledge application and transfer.

Apart from the extent and form of the linking in a hypermedia system, design considerations relating to content presentation can also influence the ultimate effectiveness of the finished system. 


\section{Content Presentation}

We know from the previous discussion that critical aspects in content presentation are the development of a coherent text structure and optimal readability. Coherence in text is assisted by the use of a well defined structure and appropriate cues to indicate this structure to the reader (van Dijk \& Kintsch, 1983). Readers find coherent and well structured text easier to read than that which is ill structured (Gillingham, 1993). Structured text provides information in a sequential fashion with elements that provide overviews and a form to which subsequent text can be added. While this structure is most often used among sentences within paragraphs and among paragraphs within sections, it also can be the framework of larger texts. The following paragraphs describe some useful strategies that can be used in developing hypermedia that lead to well structured, coherent and readable texts.

Text Structure. In hypermedia systems, text structure can be aided in a number of ways. Studies with both conventional and computer based text have found that the use of cues and overviews provide significant enhancements to structure (Thuring, Mannemann \& Haake, 1995). In hypermedia, the use of indices and tables showing the structure and relationship between nodes is a useful strategy for this. Many systems use nets to demonstrate the structure and organisation of information and to aid learners in gaining a sense of global structure. In a study that examined the impact of using overviews and document structure cues, Dee-Lucas \& Larkin (1995) found that they aided retention of information that was presented and generated a better breadth of recall of what had been read. Readers who browsed through an unstructured scrolling version of the text were found to have developed a more fragmented view of the structure of the information. The interactive overviews were a significant aid to learning.

Readability. The readability of a document is a measure of the ease with which a reader is able to comprehend what is being read. Hypermedia offers a number of ways to increase readability of the printed text. Higgins and Boone (1990) describe the following enhancements. At the surface level, difficult terms can be linked to nodes that provide further explanation and description: for example, clicking on a word to find its meaning. As an aid to increasing understanding of deeper meaning structures, the hypermedia system can be made to provide literal and inferential questions together with paragraph summaries: for example, interactive elements that cause the learners to reflect and consider that which has been read. While these forms of cues can also be provided in some ways with conventional materials, they can form a natural part of a hypermedia system readily available to those students who seek to employ them while providing no distraction to those who do not need them. 
Fragmentation. A number of studies have revealed problems emerging from learning with hypermedia caused by the fragmentation of information and learning material when it is presented as discrete elements. Fragmentation results in a lack of associative and interpretative contexts and can create a document that appears to the user as a series of discrete rather than coherent information elements. There are several ways to overcome the possibility of learners perceiving fragmentation in a hypermedia system. Most links in hypermedia serve two purposes: to show a relationship exists between two nodes and to provide a path between them. Horney (1993) suggests there should be some distinction made in these two tasks. If links could show the form of association they represent as well as providing the means to traverse, navigation through hypermedia systems would be greatly enhanced as would an associative context for linked nodes. Another strategy suggested by Thuring, Mannemann and Haake (1995) is to show new nodes in concert with their predecessor, thus establishing a coherence and semantic relationship enabling a common mental representation by the learner. Considerations of text structure in developing the content of text based nodes can also help to reduce the apparent fragmentation of stored information. Blohm (1982) found that the use of paraphrases and summaries can enhance learning. Learners using a hypertext system with this option were found to recall more information than others reading the same material without the summaries and did so in equivalent amounts of time. The summaries appeared to help to reduce the fragmentation caused by the division of the content into hypertext nodes.

Navigation and orientation. Conventional instructional materials require few operational skills on the part of the learner while hypermedia systems, being computer based, often employ many functions and features. In many cases, the functions and features of hypermedia systems can distract learners from the task at hand. It is important in designing materials to minimise the negative impact that poor interface designs can have. When learners are compelled to think and consider how an interface operates when undertaking a learning task. their attention is split and the mental effort required to attend to information from multiple sources lessens that which can be applied to the actual learning task (Chandler and Sweller, 1991). At the same time, if learners are not comfortable with the system, many advantages can be lost. Gray and Sasha (1989) report a study in which a number of learners chose not to use navigation options within a hypertext document and were wary of other features and afraid of getting lost.

There are a number of guidelines suggested by authors which can act to minimise the amount of mental and cognitive activity associated with controlling the interface. Brooks (1993) suggests a need for simplicity and consistency in design. When screens change, the only things that change 
should be the information to which the learner is being directed. Buttons and controlling features should remain in the same place and should be intuitive rather than clever in their design. Typographic clues, colour changes and unnecessary graphics all have the potential to distract and should be used sparingly. In terms of text display, distinct guidelines exist to guide hypermedia development (eg. Hartley, 1987; Wynn \& Herrington, 1995).

While hypermedia systems need simple navigation procedures, they also need to aid orientation. Orientation describes the means by which users are able to identify their current position in the system, how they achieved that position and how to return to a previous position. Disorientation is a problem which is frequently observed in studies of hypermedia users and a problem which significantly limits instructional outcomes (eg. Gay \& Mazur, 1989; Collis, 1991). Orientation is easily aided by the provision of such cues as path trails and simple graphics showing position within the net structure. These small additions to screen presentations offer significant assistance to many learners.

Text displays. As is to be expected, the actual on screen appearance of text and images from hypermedia systems influences readability. Research into graphical aspects of instructional design provides a guide for choosing how text displays should be presented and formatted. We know, for example, that students read more slowly from screens than they do from paper and reading accuracy is lessened for cognitively demanding tasks (Oliver, 1994). Readers indicate a preference for reading from high quality hardcopy rather than screen displays (Dillon, McKnight \& Richardson, 1988). While paper based instructional materials are usually confined to black text on white pages, many more variations occur in computer based learning materials. A significant amount of research has been conducted to investigate the optimal forms for screen images. Findings suggest that optimal characteristics include proportionally spaced characters, left justified, small size serif fonts for text, sans serif headings, a dense character spacing, for example, 80 characters per line, contrasting dark text on a light background (Hooper \& Hannafin, 1986).

Instructional designers frequently use graphics and images to enhance the appearance and appeal of instructional materials. It is important to make decisions concerning the usage and placement of images based on the needs of the learner. Often graphics and images that are employed solely for their aesthetic contributions can distract learners and reduce rather than enhance outcomes (Balajthy, 1990). The use of unnecessary elements in screen design extends to typography as well. The use of multiple colours, italics and various levels of headings does not appear to aid comprehension and readability and adds to the complexity of screen the design (Brooks, 1993). 


\section{Summary and Conclusions}

The use of hypermedia as an instructional medium is growing rapidly with developments and enhancements in instructional and computing technologies. Much of the popularity of the medium is derived from its capacity to convey large amounts of structured and associated information to learners in ways which promote student centred and independent learning. Recent developments in multimedia technologies and software and enhanced networking facilities through the Internet have led to new and exciting opportunities for hypermedia systems development. Whereas in previous years, hypermedia development required specific skills in software authoring, the new technologies provide the means for teachers and instructors with few skills and experience in computer based teaching to become developers and publishers of their own materials and systems.

This paper has reviewed the learning potential of hypermedia and reported on a number of studies which have demonstrated the potential to be gained from the use of this instructional medium in teaching and learning. At the same time, it has examined a number of studies which have reported problems and unanticipated outcomes arising from applications and implementations of hypermedia and hypertext systems. Analysis of outcomes from studies of hypermedia implementations suggests some key areas which need to be addressed when designing and developing effective hypermedia materials. Of particular importance are the choices relating to the forms and levels of linking that will be built into the implementation of the system, and the manner in which the content is structured and presented.

When developing hypermedia materials, it is important to be aware of the different possibilities in node structure and linking that are characteristic of the different forms which contemporary hypermedia takes. The importance of this is in being able to choose a form that is appropriate to the learning outcomes sought by the materials and to be able to cater adequately for differences among the learners with whom the system is to be implemented.

Having chosen a form for the hypermedia system, developers need to be aware of the attributes of node design and structure that can influence use and subsequent learning outcomes. Important attributes include how the textual information in nodes is structured and linked, factors influencing the readability of the stored information, strategies to make the system easy to navigate and orient within and finally considerations for designing and formatting text displays. Each of these has the potential to influence learning and can be varied to improve the learning quality of the completed system. 


\section{References}

Balajthy, E. (1990). Hypertext, hypermedia and metacognition: Research and instructional implications for disabled readers. Reading, Writing and Learning Disabilities, 6(2), 183-202.

Becker, D. \& Dwyer, M. (1994). Using hypermedia to provide learner control. Journal of Educational Multimedia and Hypermedia, 3(2), 155-172.

Blohm, P. (1982). Computer-aided glossing and facilitated learning in prose recall. In J. Niles and L. Harris (Eds.), New Inquiries in Reading Research and Instruction, pp24-28. Rochester, NY: National Reading Conference.

Brooks, R. (1993). Principles for effective hypermedia design. Technical Communication, 40(3), 422-428.

Brown, A. (1978). Knowing when, where and how to remember: A problem of metacognition. In R. Glaser (Ed.), Advances in Instructional Psychology. Hillsdale, NJ: Erlbaum.

Carroll, J. (1990). The Nurnberg Funnel: Designing minimalist instruction for practical computer skill. Cambridge, MA: MIT Press.

Chandler, P. \& Sweller, J. (1991). Cognitive load theory and the format of instruction. Cognition and Instruction, 8(4), 293-332.

Cheng, K. \& Rowe, W. (1995). Hypermedia as a tool. Computing and Control Engineering Journal, 6(3), 123-130.

Collis, B. (1991). The evaluation of electronic books. Educational and Training Technology International, 28(4), 355-363.

Dee-Lucas, D. \& Larkin, J. (1995). Learning from electronic texts: Effects of interactive overviews for information access. Cognition and Instruction, 13(3), 431-468.

Dillon, A., McKnight, C. \& Richardson, J. (1988). Reading from paper versus reading from screen. The Computer Journal, 31(5), 457-464.

Dimitroff, A. \& Wolfram, D. (1995). Searcher response in a hypertext-based bibliographic information retrieval system. Journal of the American Society for Information Science, 46(1), 22-29.

Gay, G. \& Mazur, J. (1989). Conceptualising a hypermedia design for language learning. Journal of Research on Computing in Education, 21(2), 119-126.

Gillingham, M. (1993). Effects of question complexity and reader strategies on adults' hypertext comprehension. Journal of Research on Computing in Education, 26(1), 1-15.

Glover, K. (1994). How expert systems can make hypertext more usable. Technical Communication, 41(4), 628-634.

Gray, S. \& Sasha, D, (1989). To link or not to link? Empirical guidance for the design of non-linear text systems. Behavioral Research Methods, Instruments and Computers, 21, 326-333.

Hartley, J. (1987). Designing electronic text: The role of print based research. Education Computing and Technology Journal, 35(1), 3-17. 
Heller, R. (1990). The role of hypermedia in education: A look at the research issues. Journal of Research on Computing in Education, 22(4), 431441.

Herrington, J., Fox, R., Gillard, G. \& Rainford, J. (1992). Designing Study Materials: A WADEC Guide for Authors and Desk top Publishers. Perth, WA: WADEC.

Herrington, J. \& Oliver, R. (1995). Critical characteristics of situated learning: Implications for the instructional design of multimedia for higher education. In J. Pearce \& A. Ellis (Eds.), Learning with Technology, ASCILITE'95 Conference Proceedings, pp 253-262. Melbourne: ASCILITE.

Higgins, K. \& Boone, R. (1990). Hypertext: A new vehicle for computer use in reading instruction. Intervention in School and Clinic, 26(1), 26-31.

Horney, M. (1993). Case studies of navigational patterns in constructive hypertext. Computers in Education, 20(3), 257-270.

Hooper, S. \& Hannafin, M. (1986). Variables affecting the legibility of computer generated text. Journal of Instructional Development, 9(4), 22-28.

Investigating Lake Iluka [Computer Software] (1995). Wollongong, Australia: Interactive Multimedia Pty Ltd. (FreeCall 1800500 405)

Jacobson, M. \& Spiro, R. (1995). Hypertext learning environments, cognitive flexibility and the transfer of complex knowledge: An empirical investigation. Journal of Educational Computing Research, 12(4), 301-333.

Jonassen, D. (1994). Towards a constructivist design model. Educational Technology, 34(4), 34-37.

Jonassen, D., Mayes, T. \& McAleese, R. (1993). A manifesto for a constructivist approach to uses of technology in higher education. In T. Duffy, J. Lowyck, \& D. Jonassen (Eds.), Designing Environments for Constructivist Learning, pp.231-247. Berlin Heidelberg: Springer-Verlag.

Knuth, R. \& Cunningham, D. (1993). Tools for constructivism. In T. Duffy, J. Lowyck, \& D. Jonassen (Eds.), Designing Environments for Constructivist Learning, pp.163-187. Berlin Heidelberg: Springer-Verlag.

Kunz, G., Drewniak, U. \& Schott, F. (1992). On-line and off-line assessment of self regulation in learning from instructional text. Learning and Instruction, 2, 287-301.

McGrath, D. (1992). Hypertext, CAI, paper or program control: Do learners benefit from choices? Journal of Research on Computing in Education, 24(4), 513-532.

Marchionini, G. (1989). Information-seeking strategies of novices using a full-text electronic encyclopedia. Journal of The American Society for Information Science, 40(1), 54-66.

Oliver, R. (1994). Proof-reading on paper and on screens: The influence of practice and experience on performance. Journal of Computer Based Instruction, 20(4), 118-124.

Piette, M. \& Smith, N. (1991). Hypermedia and library instruction: The challenge of design. Reference Services Review, 19(4), 13-20. 
Puntambekar, S. (1995). Helping students learn 'how to learn' from texts: Towards an ITS for developing metacognition. Instructional Science, 23(1), 163-182.

Riding, R. \& Chambers, P. (1992). CD-ROM versus textbook: A comparison of the use of two learning media by higher education students. Educational and Technology Training International, 29(4), 342349.

Small. R. \& Grabowski, B. (1992). An exploratory study of informationseeking behaviours in learning with hypermedia information systems. Journal of Educational Multimedia and Hypermedia, 1(4), 445-464.

Thuring, M., Mannemann, J. \& Haake, J. (1995). Hypermedia and cognition: Designing for comprehension. Communications of the ACM, 38(8), 57-66.

Trumbull, D., Gay, G. \& Mazur, J. (1992). Students' actual and perceived use of navigational and guidance tools in a hypermedia program. Journal of Research on Computing in Education, 24(3), 315-328.

van Dijk, T. \& Kintsch, W. (1983). Strategies of discourse comprehension. Orlando: Academic Press.

Wynn, S. \& Herrington, J. (1995). The page in print: Designing better documents with desktop publishing. Perth, WA: Edith Cowan University.

Young, M. (1995). Assessment of situated learning using computer environments. Journal of Science Education and Technology, 4(1), 89-96.

Ron Oliver has a background in instructional technologies and has lectured and researched in this area for some years. Research interests include children's information technology skills, using interactive technologies for information, instruction and training, and technology applications to support flexible and open learning, His address is Department of Library and Information Science, Edith Cowan University, Bradford St, Mt Lawley 6050, Western Australia.

Phone +61 9370 6372; fax +61 9370 2910; email r.oliver@cowan.edu.au

Jan Herrington is an instructional designer involved in the development of instructional materials for university open and flexible learning programs. She has written several books on instructional design principles for university teaching. Currently she is researching the effectiveness of different instructional design models for technology based teaching and learning.

Please cite as: Oliver, R. and Herrington, J. (1995). Developing effective hypermedia instructional materials. Australian Journal of Educational Technology, 11(2), 8-22. http:/ / www.ascilite.org.au/ajet/ajet11/oliver.html 